\title{
Eyelash in lacrimal punctum: demonstrating a negative pressure in the lacrimal sac
}

\author{
Anil Kumar Verma, Avantika Dogra, Rajeev Tuli \\ Department of Ophthalmology, Dr. Rajendra Prasad Government Medical \\ College, Kangra, Himachal Pradesh, India
}

\begin{abstract}
Eyelashes are normally arranged in two or three rows on the upper and lower eyelids. They are more numerous on the upper eyelid, with approximately 150 eyelashes being present on the upper and 75 on the lower eyelid. Eyelashes, like body hair, are shed regularly every 100 to 150 days. ' Usually, the shed eyelashes do not cause any symptoms.

However, the cilia may sometimes get misplaced and end up in the lacrimal puncta, meibomian gland orifice, subconjunctival space, and corneal stroma. An eyelash that gets misplaced into the punctum has the potential to cause additional problems. Once the eyelash enters the punctum, the barbs on the hair prevent it from being expelled. ${ }^{2}$
\end{abstract}

Keywords: canaliculus, cilium, punctum

\section{Case report}

A 41-year-old female patient presented with watery eyes, foreign body sensation, and mild erythema on the nasal bulbar conjunctiva in the left eye for last two days. She had persistent discomfort and pain in her left eye. The visual acuity in both eyes was $20 / 20$. The intraocular pressure in both eyes was normal. A slit-lamp examination revealed a protruding eyelash from the upper punctum, with the root of the eyelash into the canaliculus (Figs. 1 and 2). The conjunctiva was congested on the nasal side. Fluorescein staining revealed punctate lesions on the nasal part of cornea adjacent to the limbus. The eyelash was easily removed from the punctum with forceps. Almost $3.5 \mathrm{~mm}$ of the cilium had entered the upper punctum. She was treated with topical moxifloxacin $0.5 \%$. Corneal lesions healed within three days.

Correspondence: Dr. Anil Kumar Verma, Department of Ophthalmology, Dr. Rajendra Prasad Government Medical College, Kangra, Himachal Pradesh 1760012, India.

E-mail: anilkumarverma2971@gmail.com 


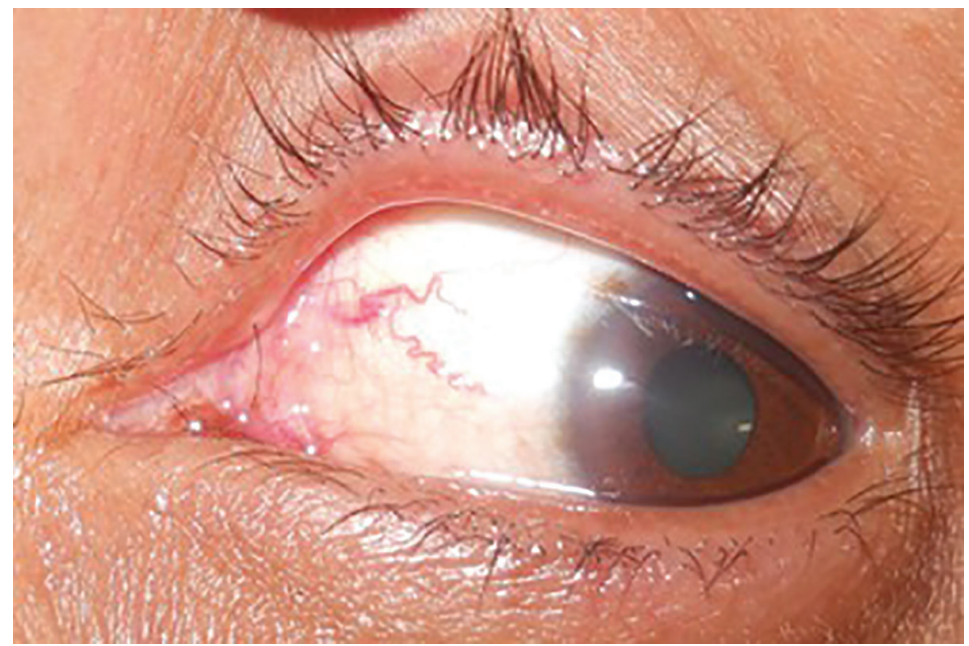

Fig. 1. Outer segment of the left eye with protruding cilium from the upper punctum.

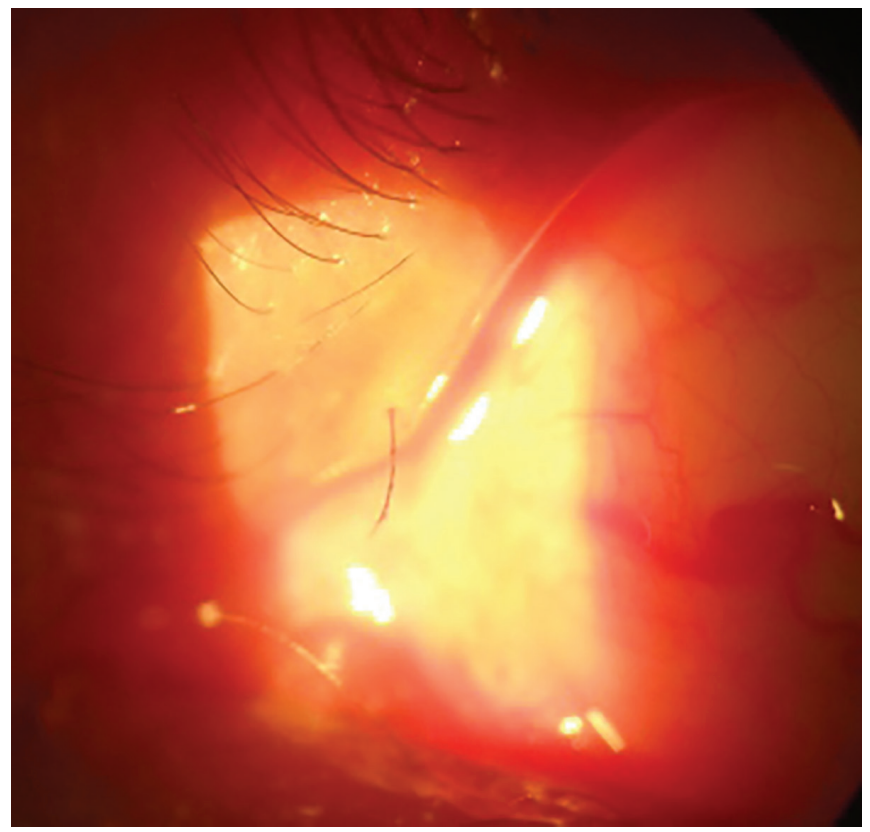

Fig. 2. Slit-lamp photograph showing nasal bulbar erythema with cilium protruding from the upper punctum. 


\section{Discussion}

The presence of eyelash in a lacrimal punctum is said to be a not infrequent occurrence, although many authoritative textbooks do not mention it. ${ }^{3}$ Boase $^{3}$ stated that, anatomically, the cilium has no business lodging itself in this peculiar position. The average length of the vertical portion of the canaliculus is 1.8 to $2.25 \mathrm{~mm}$. There is a physiological constriction just above the punctum where the canaliculus is 0.1 $\mathrm{mm}$ wide, much less than the average cilium. The eyelash would have fallen out when the patient rubbed her eyes, and its root lying in front of the punctum would have been sucked into the canaliculus, thus demonstrating a negative pressure in the lacrimal sac as postulated by several theories of the conduction of tears. ${ }^{4}$ Once an eyelash is shed into the external ocular surface, it can cause foreign body sensation, leading to reflex tearing that will carry it away to the lacus lacrimalis where it can be propelled by lids or sucked into the canaliculus in the blink cycle. ${ }^{5}$

Unusual location of cilia after falling out of their follicles may cause diagnostic difficulty. Gutteridge ${ }^{6}$ and Agarwal et al. ${ }^{7}$ reported cases of cilium lodging in the orifice of the meibomian gland. Jain et al. ${ }^{8}$ reported a trimmed eyelash embedded in the meibomian gland orifice, which simulated the symptoms of endophthalmitis after an uneventful phacoemulsification, and it was suggested that trimming of eyelashes should be avoided preoperatively. Eyelashes are reported to enter the upper punctum more frequently than the lower punctum. Werb ${ }^{9}$ and Meel and Vashisht ${ }^{2}$ reported cases of eyelash impacted in the upper lacrimal punctum. Yeo ${ }^{10}$ reported a female patient who presented with a cut hair end lodged in the upper punctum. Her history revealed that she had a haircut earlier that day after which her symptoms started. Nagashima and Kido"11 studied 88 cases of impaction of eyelash in the lacrimal punctum, and they found that cilia in the upper punctum were three times more frequent than cilia in the lower punctum.

To conclude, in patients with nonspecific eye symptoms like irritation, watering, mild erythema of nasal bulbar conjunctiva, etc., a misplaced eyelash may be the cause, which can be easily overlooked and treated inappropriately. Therefore, it is important to examine the eyelid margin carefully in such cases.

\section{References}

1. Snell RS, Lemp MA. Clinical Anatomy of the Eye. Boston, MA: Blackwell Scientific Publications; 1989:9.

2. Meel R, Vashisht S. Eye lash in lacrimal punctum. Delhi J Ophthalmol. 2013;23:227.

3. Boase AJ. Eye lash in lacrimal punctum. Br J Ophthalmol. 1949;33:513.

4. Stern JJ. Eye lash in upper lacrimal punctum. Am J Ophthalmol. 1952;35:1206.

5. Ziahosseini K, Odat TAM. 5 cases, 1 cause of irritated eyes. J Fam Pract. 2007;56:365-368.

6. Gutteridge IF. Curious cilia cases. Clin Exp Optom. 2002;85:306-308.

7. Agarwal S, Agarwal J, Agarwal TP. Cilium as a foreign body in a meibomian gland opening. J Cataract Refract Surg. 2003;29:1047. 
8. Jain AK, Sukhija J, Vinekar A, Chopra I, Gupta A. Loose cilium in meibomian gland: mimicking early postoperative endophthalmitis. Eye. 2006;20:957-958.

9. Werb A. Unusual causes of epiphora. Br J Ophthalmol. 1971;55:559.

10. Yeo DCM. An unusual foreign body in eye. Br Med J Case Rep. 2013. doi:10.1136/ bcr-2013-200144.

11. Nagashima K, Kido R. Relative roles of upper and lower lacrimal canaliculi in normal tear drainage. Jpn J Ophthalmol. 1984;28:259-262. 